\title{
Topological Magnetic States and their Properties
}

Sergio A. Montoya

Space and Naval Warfare Systems Center Pacific, San Diego, CA, USA.

Skyrmions are topologically non-trivial particle-like magnetic spin textures that exhibit novel physics and potential technological applications [1-3]. These spin textures are usually stabilized by Dzyaloshinskii-Moriya interaction (DMI) that arises in non-centrosymmetric bulk magnets or thin ferromagnetic film heterostructures with asymmetric transition metal interfaces that possess high-spin orbit coupling. Topologically equivalent magnetic spin textures can also be stabilized by the competition of dipole energy and domain wall energy, termed dipole skyrmions [4]. A skyrmion is characterized in terms of a topological number $S$, defined as: $S=\frac{1}{4 \pi} \int M$. $\left(\partial_{x} M \times \partial_{y} M\right) d x d y$. The topological nature of a skyrmion enables some of its unique properties, such as: current-driven motion with low current densities, topological Hall effect, skyrmion Hall effect, localized non-trivial spin wave dynamical modes resulting from microwave perturbations, among other characteristics.

This talk focuses on recent developments in stabilizing, manipulating and designing topologically non-trivial magnetic states in thin film heterostructures. Using a combination of real- and reciprocal-space measurements, such as Lorentz transmission electron microscopy, full-field transmission soft x-ray microscopy and resonant soft x-ray scattering, we demonstrate the formation of various topological magnetic spin structures such as achiral bubbles $(S=0)$, skyrmions $(S=1)$, bi-skyrmions $(S=2)$ [5] and anti-skyrmions $(S=-1)$ which arrange in either closed-packed lattice or isolated magnetic phases. Emphasis is given to dipole skyrmions $(S=1)[4]$ and their properties.

First, we show that sub-100nm dipole skyrmions are achievable in magnetic specimens where the thin-film shape anisotropy $2 \pi M_{S}^{2}$ exceeds the uniaxial anisotropy $K_{U}$. The ratio $Q=K_{U} / 2 \pi M_{S}^{2}$, commonly referred as a material's $Q$-factor is $<1$. A dipole skyrmion exhibits a complex spin structure that varies across the magnetic specimen thickness: along the center of the slab it exhibits a Bloch-like wall configuration that broadens and transitions to Néel-like walls towards the film surface which are often referred to as closure domains or Néel caps (Fig. 1). Unlike DMI skyrmions, a dipole skyrmion phase consists of an equal population of chiral cylindrical-like spin textures with two possible helicities such that, on average, the material is achiral.

Numerical simulations of the Landau-Lifshitz-Gilbert (LLG) equation, utilizing the FastMag solver, enables us to explore the magnetic energy parameter space to determine the different ratios of magnetization $M_{S}$, uniaxial anisotropy $K_{U}$ and exchange interaction $A$ that result in formation of dipole skyrmions. We show that proper combinations of $\left(M_{S}, K_{U}, A\right)$ can result in dipole skyrmion phases for a slab of a given thickness, and we observe dipole skyrmion feature size can be correlated to the magnetic slab thickness. Experimentally, we demonstrate dipole skyrmions with features down to $20-\mathrm{nm}$ are achievable utilizing highly tunable magnetic properties of amorphous $\mathrm{Fe} / \mathrm{Gd}$ multilayer films. 
We also demonstrate deterministic current-induced displacement of sub-100nm dipole skyrmions by spin-transfer effect [6]. Experiments are performed on amorphous $\mathrm{Fe} / \mathrm{Gd}$ multilayers that are patterned into wires and exhibit stripe domains and dipole skyrmions at room temperature. The magnetic textures exhibit motion under current excitations with a current density $\sim 10^{8} \mathrm{~A} / \mathrm{m}^{2}$. We present current-induced dynamics for different magnetic phases, including disordered stripe domains, coexisting stripes and dipole skyrmions and a closed packed dipole skyrmion lattice.

\section{References:}

[1] N Nagaosa and Y Tokura, Nature Nanotechnology 8, (2013), p. 899-911.

[2] A Fert, N Reyren and V Cros, Nature Reviews 2, 17031 (2017).

[3] W Jiang et al, Physics Reports 704, (2017), p. 1-49.

[4] S A Montoya et al, Physical Review B 95, 024415 (2017).

[5] J C T Lee et al, Applied Physics Letters 109, 022402 (2016).

[6] S A Montoya et al, Preprint (2018).
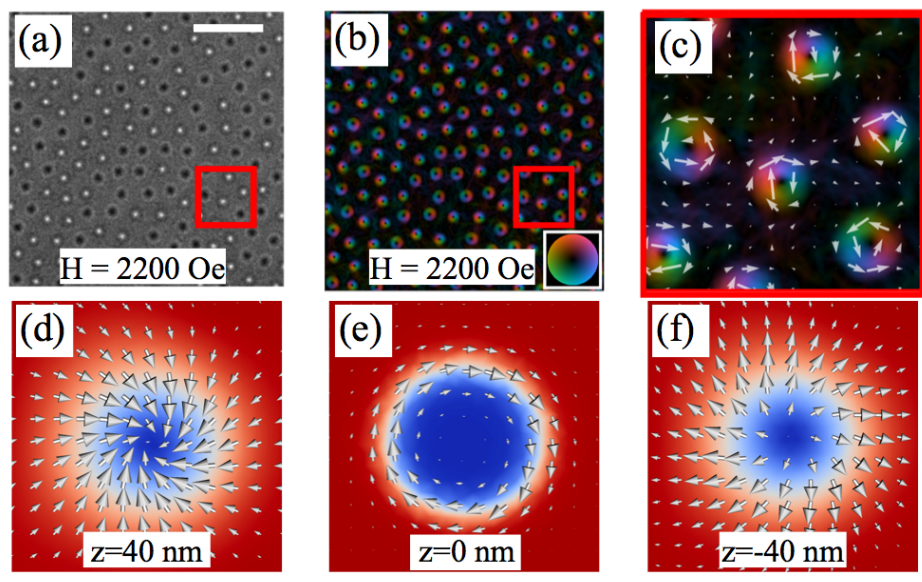

Figure 1. (a-c) Lorentz TEM image of a closed packed lattice of dipole skyrmions in an 80-nm thick Fe/Gd film and (d-f) Numerical simulations of a single dipole skyrmion $(\boldsymbol{S}=\mathbf{1}, \boldsymbol{\gamma}=-\boldsymbol{\pi} / \mathbf{2})$ at different depths for an 80-nm thick slab with properties $M_{S}=400 \mathrm{emu} / \mathrm{cm}^{3}, K_{U}=4 \times 10^{5} \mathrm{ergs} / \mathrm{cm}^{3}$, $A=5 \times 10^{-7} \mathrm{erg} / \mathrm{cm}$. The bar in (a) is $1 \mu \mathrm{m}$. Figure is adapted from results in [4]. 